\title{
An Echo of Consciousness: Brain Function During Preferred Music
}

\author{
Manon Carrière, ${ }^{1,2}$ Stephen Karl Larroque, ${ }^{1,2}$ Charlotte Martial, ${ }^{1,2}$ Mohamed Ali Bahri, ${ }^{3}$ Charlène Aubinet, ${ }^{1,2}$ \\ Fabien Perrin, ${ }^{4}$ Steven Laureys, ${ }^{1,2, *}$ and Lizette Heine ${ }^{4, *}$
}

\begin{abstract}
Background: Given its emotional and autobiographical properties, music appears as a potential aid in diagnostic assessment and therapeutics in patients with disorders of consciousness (DOCs). Several studies have shown boosting effects on behavior and brain functioning when patients were exposed to (their preferred) music.

Objective: The aim of this study was to investigate if these effects on the brain's spontaneous activity are dependent on the level of consciousness.

Methods: Nine postcomatose patients and eight healthy control subjects were assessed using functional magnetic resonance imaging in two conditions: rest (without stimulation) and music. All patients presented at least an auditory startle, thereby suggesting the presence of residual auditory function. A gradient of consciousness (from unresponsive wakefulness syndrome to healthy subjects) was formulated to evaluate consciousness-level-dependent increases in brain activity and connectivity. Network-based functional connectivity assessed auditory, default-mode, frontoparietal, and music-evoked emotion networks. Furthermore, exploratory voxel-to-voxel analyses were performed at the whole brain level using intrinsic connectivity contrast and (fractional) amplitude of low-frequency fluctuations.

Results: Stronger consciousness-level-dependent increases within network-to-voxel analysis of connectivity were found in the frontoparietal network with the precuneus during music stimulation compared with rest. Voxel-to-voxel analyses showed stronger increases of intrinsic connectivity in the music condition compared with rest in regions previously related to music processing. There were consistently more regions with increased connectivity during the main effect of music compared with rest. These increases of connectivity during music were observed in brain regions involved in consciousness, language, emotion, and memory processing.

Conclusions: Our results show that music seems to trigger more substantial connectivity changes compared with rest, pointing toward the potential ability of music to stimulate patients' brain function. Further research should focus on effects of music in general, its specific acoustical features, and the effects of simple auditory stimuli, as well as the possible therapeutic and diagnostic effects of music in well-controlled clinical trials.
\end{abstract}

Keywords: disorders of consciousness; (fractional) amplitude of low-frequency fluctuation; functional connectivity; MRI; music

\section{Impact Statement}

Due to its emotional and autobiographical properties, music is a particularly salient stimulus. A few studies using musical stimuli have shown promising results in terms of behavioral responsiveness in patients with disorders of consciousness (Magee, 2005; Raglio et al., 2014; Verger et al., 2014). Our study confirms the presence of a beneficial effect of music on brain connectivity in these severely brain-injured patients, which moreover seems to be dependent on the level of consciousness. Our findings therefore strengthen the hypothesis that music could play a role from a diagnostic and therapeutic standpoint in this population, paving the way for future well-controlled clinical trials.

\footnotetext{
${ }^{1}$ Coma Science Group, GIGA-Consciousness, University of Liège, University Hospital of Liège, Liège, Belgium.

${ }^{2}$ Centre du Cerveau, University Hospital of Liège, Liège, Belgium.

${ }^{3}$ GIGA-Cyclotron Research Center In Vivo Imaging, University of Liège, Liège, Belgium.

${ }^{4}$ Lyon Neuroscience Research Center, Auditory Cognition and Psychoacoustics Team, Inserm U1028—CNRS UMR522, University of Lyon1, Lyon, France.

*Both authors share the last position.
} 


\section{Introduction}

D ISORDERS OF CONSCIOUSNESS (DOCs) remain a very challenging pathological condition. After emerging from coma, severely brain-injured patients may pass through several altered states of consciousness. In the unresponsive wakefulness syndrome (UWS-The Multi-Society Task Force on PVS, 1994), patients are awake but show no signs of awareness of themselves or their environment. Once they demonstrate unequivocal (but often inconsistent) behavioral evidence of self or environmental awareness, they are said to be in a minimally conscious state (MCS - Giacino et al., 2002). Some patients present a cognitive motor dissociation, demonstrating consciousness using novel neuroimaging techniques while being unresponsive at the bedside (Schiff, 2015). Patients emerge from MCS (EMCS) when they regain functional communication or objects use (Giacino et al., 2002).

Besides, patients suffering from locked-in syndrome (LIS) are frequently mistakenly diagnosed as unconscious due to motor paralysis, which prevents them from expressing their consciousness and cognitive abilities (Schnakers et al., 2008). The ability to manifest consciousness is indeed jeopardized by such limited motor responses, but also by significant cognitive and sensory impairments, as well as vigilance fluctuations (Schnakers et al., 2009). Consciousness is thus difficult to assess in these noncommunicative patients, and repeated assessments by trained clinicians are needed (Wannez et al., 2017a) to provide a reliable and accurate diagnosis, which crucially influences important medical and therapeutic decisions (Boly et al., 2008; Demertzi et al., 2011).

There is some indication that the use of salient or autobiographical stimuli could optimize arousal and allow more accurate diagnoses (Perrin et al., 2015). DOC patients would indeed be more responsive when assessed with personally salient versus neutral stimuli. Improved scores on the most sensitive standardized behavioral assessment scales (Coma Recovery Scale-Revised; CRS-R_Giacino et al., 2004) can be seen with, for example, self-related objects to evaluate functional use of objects (Sun et al., 2018), a mirror to assess visual pursuit (Wannez et al., 2017b) and the own name to evaluate localization to sound (Cheng et al., 2013).

Music has specifically received special attention in recent literature (for a review, see Grimm and Kreutz, 2018), as music can be very personal. For example, higher CRS-R scores and more frequent spontaneous signs of consciousness are observed after preferred music exposure (Verger et al., 2014). Using electroencephalography, exposure to preferred music increases the probability to observe a $\mathrm{P} 300$ response to one's own name in DOC patients (Castro et al., 2015). Authors hypothesize that this is due to its emotional and self-related properties. Furthermore, several case studies have so far reported potential long-term effects of music therapy on behavioral responses (e.g., increased eye contacts and smiles, reduction of annoyance, inertia, or psychomotor agitation) (Magee, 2005; Raglio et al., 2014).

The search for more reliable diagnoses often uses neuroimaging to assess residual brain function. Resting-state functional magnetic resonance imaging (fMRI) is one such tool and allows for the exploration of several reproducible brain networks without the need for patients' cooperation. In DOCs, this technique has proven to be a valuable tool to complement the behavioral assessment as it shows a decrease in functional connectivity (Heine et al., 2012) and can distinguish between UWS and MCS patients (Demertzi et al., 2015). Such a linear relation between functional connectivity and consciousness in resting-state networks can be extended to EMCS patients (Di Perri et al., 2016). Notably, the auditory network has been shown with the strongest power to distinguish between UWS and MCS, allowing for automatic classification (Demertzi et al., 2015).

The first fMRI study on music in DOCs found an activation in auditory regions for healthy control subjects (HCSs) and MCS patients, as well as in one UWS patient who would soon evolve into MCS (Okumura et al., 2014). Another study showed that within patients, changes in functional connectivity might be related to the auditory network implied in rhythm and music perception, and to cerebral regions within the frontoparietal network (FPN) linked to autobiographical memory (Heine et al., 2015). The findings that music "boosts" cognitive functions lead to the hypotheses that salient sensory stimuli might temporarily bring the patients' brain function to its full potential, which can thus improve diagnostic assessment.

Within this study, we aim to assess if these observed effects of music on the brain's spontaneous activity are dependent on the level of consciousness. To do so we formulated a gradient of consciousness (from UWS to HCS), and we explored functional connectivity (with a priori network-based analysis and exploratory voxel-to-voxel analysis) and spontaneous regional brain activity ([fractional] amplitude of low-frequency fluctuation-fALFF) changes during a music and a rest condition. We expect to observe a stronger linear correlation with the level of consciousness during music.

\section{Materials and Methods}

\section{Participants}

Eight HCSs (four females; mean age $=26$ years, standard deviation $[\mathrm{SD}]=3$; Table 1) and 12 patients (4 UWS, 2 MCS, 3 EMCS, 3 LIS) were included in the study between March 2014 and April 2018. All the HCSs and five of the 12 patients were the same as in a previous study (Heine et al., 2015). Inclusion criteria for HCS were the absence of history of neurological or psychiatric disorders. Inclusion criteria for patients were as follows: (1) prolonged DOC or LIS (>1 month postinjury); (2) absence of contraindications for MRI (e.g., presence of ferromagnetic aneurysm clips, pacemakers); (3) absence of sedation in the scanner; (4) absence of extensive brain damage making the analyses impossible (i.e., $>2 / 3$ of 1 hemisphere); and (5) multiple behavioral assessments using the CRS-R performed by experienced clinicians (except for LIS patients), including one on the same day before the MRI acquisition. The study was approved by the ethics committee of the medical school of the University of Liège.

\section{Protocol and data acquisition}

The protocol and data acquisition were performed as previously described (Heine et al., 2015). Five excerpts of preferred musical pieces were selected from a questionnaire completed by the participants themselves (for the HCS) and by the loved ones (for the patients), and combined to 
Table 1. Demographic and Clinical Data of the Severely Brain-Injured Patients Included in the Study

\begin{tabular}{|c|c|c|c|c|c|c|}
\hline & Gender & $\begin{array}{l}\text { Age } \\
\text { (years) }\end{array}$ & $\begin{array}{l}\text { Time since } \\
\text { injury } \\
\text { (months) }\end{array}$ & Etiology & Major structural lesions & Best $C R S-R$ score \\
\hline UWS1 & Male & 40 & 11 & Trauma & $\begin{array}{l}\text { Diffuse axonal lesions in subcortical WM, } \\
\text { temporal poles, and hippocampi. Gliotic } \\
\text { lesions in the thalami, left pallidum, right } \\
\text { caudate nucleus, and in the juxtacortical } \\
\text { regions of WM in frontal lobes }\end{array}$ & A1 V0 M2 O1 C0 Ar1 \\
\hline UWS2 & Female & 50 & 6 & Anoxia & $\begin{array}{l}\text { Extended cortical atrophy in frontomedian, } \\
\text { frontoparietal, and insulotemporal regions. } \\
\text { Subcortical atrophy with enlargement of } \\
\text { lateral ventricles }\end{array}$ & A1 V0 M2 O1 C0 Ar2 \\
\hline UWS3 & Male & 24 & 12 & Trauma & $\begin{array}{l}\text { Diffuse and severe cortical and subcortical } \\
\text { atrophy }\end{array}$ & A1 V1 M2 O1 C0 Ar1 \\
\hline MCS1 & Female & 39 & 42 & Trauma & $\begin{array}{l}\text { White and gray matter damage in the right } \\
\text { frontoinsular region, mesiofrontal cortex, } \\
\text { thalamus, basal ganglia, and hippocampus }\end{array}$ & A2 V3 M2 O2 C0 Ar1 \\
\hline EMCS1 & Female & 43 & 4 & Hemorrhage & $\begin{array}{l}\text { Atrophy of dorsal and frontal areas and } \\
\text { ventricles hypertrophia }\end{array}$ & A3 V1 M5 O3 C2 Ar2 \\
\hline EMCS2 & Male & 40 & 5 & Ischemia & Subcortical atrophy involving left thalamus & A4 V5 M6 O2 C2 Ar3 \\
\hline EMCS3 & Male & 60 & 13 & Stroke & $\begin{array}{l}\text { Extended tissue damage in the posterior } \\
\text { cerebral artery territory involving the } \\
\text { thalamus, and enlargement of the right } \\
\text { temporal horn of the lateral ventricle }\end{array}$ & A4 V5 M6 O3 C2 Ar3 \\
\hline LIS1 & Female & 65 & 84 & Stroke & $\begin{array}{l}\text { Malacic vascular lesion involving a large } \\
\text { part of the protuberance and the upper part } \\
\text { of the cord. Focal hyperintense frontobasal } \\
\text { focal lesions. Cerebellum atrophy }\end{array}$ & N/A \\
\hline LIS2 & Male & 50 & 5 & Stroke & $\begin{array}{l}\text { Gray matter damage in the cerebellum, } \\
\text { hypothalamus, ventromedial cortex, } \\
\text { sensorimotor cortex, insula, temporal lobe, } \\
\text { and basal ganglia }\end{array}$ & N/A \\
\hline
\end{tabular}

CRS-R, Coma Recovery Scale-Revised; EMCS, emerge from MCS; LIS, locked-in syndrome; MCS, minimally conscious state; N/A, non applicable; UWS, unresponsive wakefulness syndrome; WM, white matter.

create a musical stimulus over the duration of the functional MRI sequence. A 3 T tesla (Siemens Magnetom TrioTim) was used at the University Hospital Center of Liège. A high-resolution T1-weighted image was acquired, as well as T2*-weighted fMRI images (300 volumes, 32 slices, voxel size $=3.0 \times 3.0 \times 3.75 \mathrm{~mm}^{3}$, repetition time $=2000 \mathrm{~ms}$, echo time $=30 \mathrm{~ms}$, flip angle $=90^{\circ}$, field of view $=384 \mathrm{~mm}$, matrix size $(I, J, K)=64 \times 64 \times 32$, delay $=0$, slice order $=$ sequential descending, Echo Planar Imaging sequence with axial slice orientation). Two functional scans were acquired during one MRI scanning session: one with the participants' preferred music (i.e., music condition) and the other when participants were exposed only to the noise of the scanner (i.e., rest condition). These two functional scans were always separated by a period of $10 \mathrm{~min}$ to reduce any potential order effects, and the order of the conditions was randomized between participants. We delivered instructions and music through MR-compatible Siemens headphones. Both HCSs and patients were asked to keep their eyes closed and not think of anything in particular (compliance of patients cannot be guaranteed due to their condition).

\section{Data Preprocessing}

\section{Structural imaging}

Structural ( $11^{*}$-weighted) MRI images were manually reoriented to the anterior commissure/posterior commissure scheme and segmented into gray matter (GM), white matter (WM), and cerebrospinal fluid, and normalized into the Montreal Neurological Institute (MNI) space, using the old segmentation module and standard tissue probability map of Statistical Parametric Mapping 12 (SPM12) (Statistical Parametric Mapping 12, n.d.) under MATLAB R2011A.

\section{Resting-state fMRI}

Functional volumes were manually reoriented into the corresponding structural images, and then preprocessed by using SPM12. First, the EPI volumes were corrected for the temporal difference in acquisition among different slices using the slice timing correction module with the reference slice set to the first temporal slice, and then the images were realigned to the first volume for head-motion correction. The mean BOLD image across all realigned volumes was then coregistered with the structural image. The normalization transformation from subject space to MNI space was then applied to the coregistered BOLD images, hence applying an indirect normalization. Finally, all the coregistered BOLD images were smoothed ( $8 \mathrm{~mm}$ full-width half-maximum).

To reduce loss of data or subject exclusion due to motion artifacts, we used the artifact detection toolbox (NITRC ART) for artifact detection and rejection using a composite motion measure (largest voxel movement) with a "liberal" threshold (global threshold 9.0, motion threshold 2.0, use 
scan-to-scan motion and global signal). With this approach, a volume was defined as an outlier (artifact) if the largest voxel movement detected was above the specified thresholds. We subsequently included outliers in the global mean signal intensity and motion as nuisance regressors (i.e., one regressor per outlier in the first-level general linear model). Thus, the temporal structure of the data was not disrupted.

Several parameters were included in a linear regression using CONN v17F (Whitfield-Gabrieli and NietoCastanon, 2012) and SPM12 to remove possible spurious variances from the data. These were (1) six head-motion parameters obtained in the realigning step, along with their first-order derivatives; (2) the scrubbed BOLD volumes with too much motion as detected with the ART toolbox's composite motion measurement with a liberal setting; (3) non-neuronal noise signal derived by the anatomical component-based noise correction method (aCompCor-Behzadi et al., 2007), which defines noise signal as the top five principal components from subjectspecific WM and the top five from cerebral spinal fluid; (4) the effect of each session's start to reduce the impact of MRI magnetization oversaturation; and (5) a linear detrending to the overall resulting BOLD signal. Then, the residual time series were linearly detrended (no despiking) and temporally band-pass filtered $(0.008-0.09 \mathrm{~Hz})$ using CONN's denoising procedure.

The data that support the findings of this study are available from the corresponding author upon reasonable request.

\section{Statistical Analysis}

\section{Resting-state fMRI}

We conducted the analysis of the fMRI resting-state functional connectivity through the CONN v17F toolbox under MATLAB R2017A by using two types of analysis: (1) a seed-based correlation analysis approach, more specifically network-to-voxel to cover changes in correlation of the BOLD signal in the whole brain with respect to the specified seed regions; and (2) voxel-to-voxel connectivity measures without a priori seeds, to analyze globally the brain connectivity patterns.

For both seed-based and exploratory voxel-to-voxel analyses, second-level statistical analyses were performed to make between-groups and between-conditions comparisons. Regarding the between-groups comparison, we created a contrast that assumes a linear correlation between the state of consciousness and the connectivity's z-values: $(-3-113)$ for, respectively, the UWS, MCS and EMCS, LIS and HCS (with the MCS and EMCS patients merged in one single group). This contrast was specifically designed to produce a linear regression with a constant slope of two between our four groups. Regarding the between-conditions comparison, three different contrasts were used: main effect of the rest condition ([1 0$]$ ), main effect of the music condition ([0 1$]$ ), and a paired $t$-test difference between both conditions to highlight the effect of music compared with rest $([-11]$, or music $>$ rest $)$. In short, a significant positive correlation with the level of consciousness in music $>$ rest means that conscious subjects display increases in positive correlations compared with less conscious states that are more marked during music than rest.
We showed brain connectivity if the main effect contrast (i.e., under a single condition) or the difference in correlation between conditions effect size allows to disambiguate the conditions.

Network-to-voxel analysis. Using CONN, we used extracts from fMRI BOLD time series from a region of interest (the seed) and measured the temporal correlation between this signal and the time series of all other brain voxels. This process was repeated for each subject and each region of interest. We investigated the following networks, as composed of a set of regions of interests using an average effect contrast (i.e., uniformly weighted contrast, equivalent as to having one region of interest covering the entire network): the default-mode (DMN) and the FPN, derived from the Harvard-Oxford atlas provided by CONN. We also assessed the auditory network using a $10 \mathrm{~mm}$ radius sphere around coordinates as defined in previous studies (Demertzi et al., 2015). Furthermore, using the coordinates provided by a meta-analysis of 21 previous studies, we explored a "music-evoked emotions" network using a $5 \mathrm{~mm}$ radius sphere around coordinates (Koelsch, 2014). The regions of interest composing each network and their coordinates are detailed in Supplementary Table S1.

Exploratory voxel-to-voxel analyses. We examined the global brain connectivity patterns, without any a priori seed, between each voxel and the rest of the brain using two measures. First, the intrinsic connectivity contrast (ICC; in CONN toolbox), which permits us to observe "the sum of the correlation of each source voxels to every other voxel in the brain, which is then weighted by the root mean square of the correlation coefficient values associated with the voxel-to-voxel connection'; second, the ALFF and fractional ALFF (fALFF; present in the CONN toolbox), which are not connectivity measures per se, but thought to reflect cortical excitability and long-range neural synchronization of spontaneous neuronal activity. They can be seen as measures of the variation in the amplitude of the BOLD signal, by calculating the power in the very low frequencies, usually $0.01-0.08 \mathrm{~Hz}$, hence complementing connectivity analyses, which measure synchrony. In this study, we used a band-pass filter around the $0.008-0.09 \mathrm{~Hz}$ range for all BOLD analyses, which is the default for connectivity analyses in CONN, hence allowing better comparability between measures. The main difference between ALFF and fALFF measures lies in the resilience of the latter to the noisy fluctuations from large blood vessels, and in more robust measures regardless of head motion, although we here applied the same preprocessing as for connectivity analyses, which should reduce the impact of motion on ALFF as suggested in previous studies (Turner et al., 2013).

Statistical results were generated with CONN $17 f$ and considered significant with multiple comparison correction at the topological level with nonparametric permutation test cluster-mass $p$-false discovery rate $<0.05$ and with primary voxel-wise threshold $p$-uncorrected $<0.001$.

\section{Results}

\section{Quality control assessment}

Patients' lesions were accounted for (1) directly by excluding patients with extensive brain lesions, which would 
either prevent proper segmentation or may impair the inference (three patients were discarded for this reason); (2) indirectly by relying on aCompCor denoising, which ensures we derive most of the signal used for inference from the neuronal components in the signal from segmented GM, while regressing non-neuronal signal from non GM tissues, which indirectly includes lesions. For the latter to work as expected, we ensured with visual inspection that there was no anomaly in the segmentation and normalization of the GM of all the included subjects. Supplementary Figures S1 and $\mathrm{S} 2$ provide figures of the original $\mathrm{T} 1$ and segmented GM for all participants and for the three excluded patients.

Neither patients nor HCSs showed a significant difference in the number of motion outliers between music and rest conditions [two-sided paired $t$-test; patients: $p=0.42$, music mean $\pm \mathrm{SD}=14 \pm 14$, median (range) $=11(46)$; rest mean \pm $\mathrm{SD}=9 \pm 9$, median (range) $=7$ (31); HCS: $p=0.31$, music mean $\pm \mathrm{SD}=23 \pm 17$, median (range) $=21$ (54); rest mean \pm $\mathrm{SD}=19 \pm 19$, median (range) $=13$ (51)]. No significant difference was found between patients and HCSs for the rest $(p=0.20)$ and for the music condition $(p=0.21)$. The highest observed percentage of motion outliers was 18.3 in one HCS and 15.6 in one patient. The percentage of motion outliers related to the totality of volumes per subject and session can be found in Supplementary Table S2. Finally, the histograms of connectivity provided by CONN at the denoising step are available in Supplementary Figure S3.

\section{Network-to-voxel analysis of consciousness-level- dependent changes in connectivity}

Within the music condition, consciousness-level-dependent increases of connectivity were found in the FPN and in the DMN (Fig. 1 and Supplementary Table S3). In the FPN, such increases of connectivity were found with four clusters covering (1) the left angular gyrus, (2) the left frontal pole, (3) the right middle frontal gyrus, and (4) the right frontal pole. In the DMN, we identified consciousness-level- dependent increases of connectivity with three clusters covering (1) the left lateral occipital cortex, (2) the left posterior cingulate gyrus/precuneus, and (3) the left inferior frontal gyrus (pars orbitalis). No significant consciousness-level-dependent increase of connectivity was found for the auditory network (with or without visual areas) and music-evoked emotions network.

During rest, consciousness-level-dependent changes in connectivity were only observed within the DMN (Fig. 1 and Supplementary Table S3). This network showed an increase of connectivity with three clusters covering (1) the left posterior cingulate gyrus/precuneus, (2) the left hippocampus, and (3) the right middle temporal gyrus.

Looking at effect sizes of both conditions separately, we observe a linear gradation of the connectivity increase along with the level of consciousness (from (E)MCS to HCS), except for the group of UWS who appears to present a hyperconnectivity (Supplementary Fig. S4). This linear gradation is most marked for the FPN.

When comparing the music with the rest condition, consciousness-level-dependent changes in connectivity were found for the FPN with one cluster located in the precuneus/PCC (Fig. 2a and Supplementary Table S3). No significant difference between the two conditions was observed for any of the other networks.

Looking at effect sizes, we observe that the difference of connectivity between the two conditions seems to be driven by the group of (E)MCS, while the other groups remain constant (Fig. 2b).

\section{Voxel-to-voxel analysis of consciousness-level-dependent changes}

Intrinsic connectivity. During the music condition, analysis revealed a consciousness-level-dependent increase of intrinsic connectivity (Fig. 3a and Supplementary Table S4) in seven clusters covering (1) the left lateral occipital cortex, left angular gyrus, and left supramarginal gyrus; (2) the

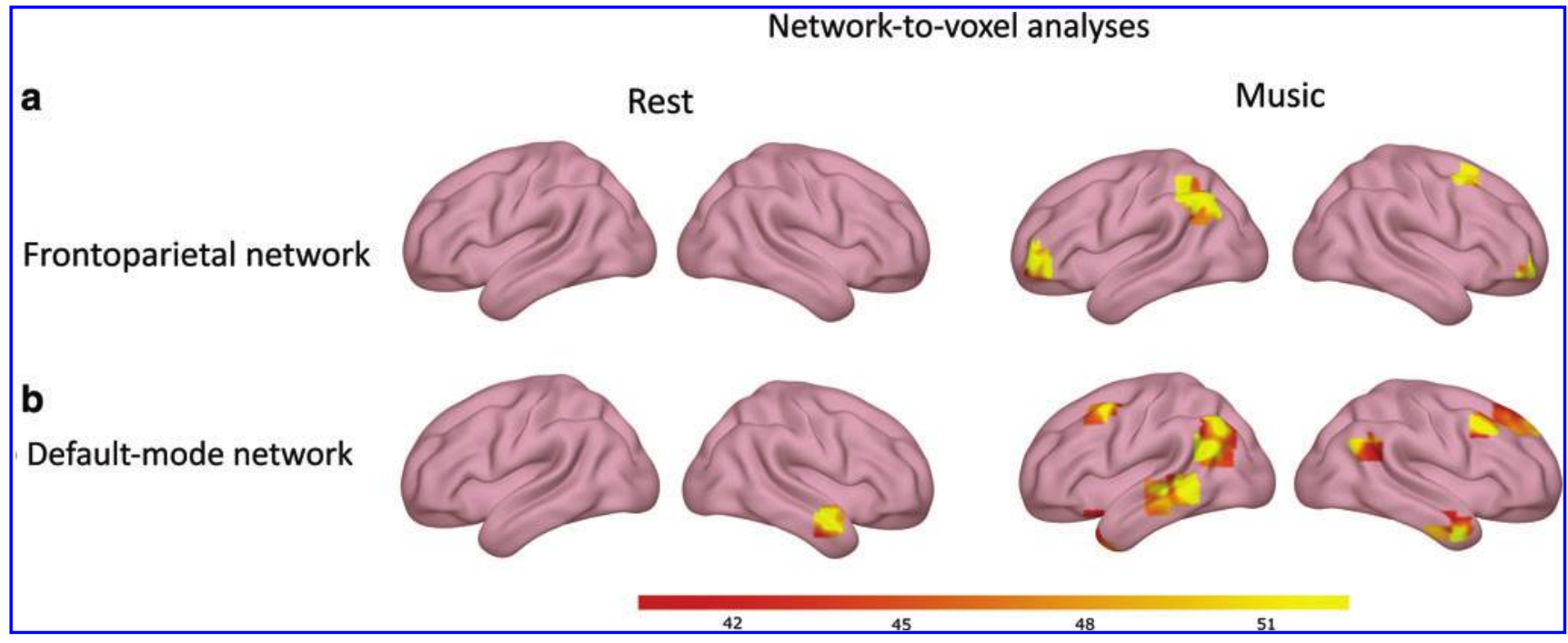

FIG. 1. Mean network connectivity in patients during the rest condition (on the left) and during the music condition (on the right) for the FPN (a) and DMN (b). Maps show the regions presenting consciousness-level-dependent increases of connectivity. Color bar indicates the strength of the connectivity. DMN, default-mode network; FPN, frontoparietal network. Color images are available online. 


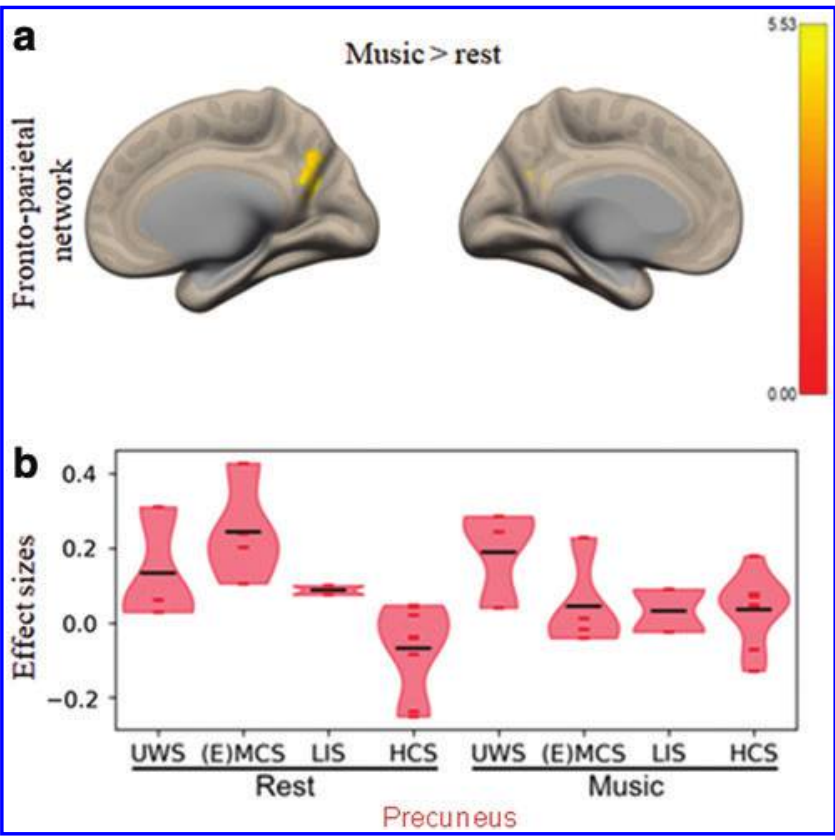

FIG. 2. (a) Mean network connectivity in patients during the music compared with the rest condition for the FPN. Maps show the regions with stronger consciousness-leveldependent increases of connectivity. (b) Effect sizes ( $z$-values) for the cluster (precuneus) showing increased correlation across consciousness states with the FPN in the music compared with the rest condition. The violin plots represent kernel density estimation. Each red dot represents one patient/ healthy subject and black lines represent the mean. Color images are available online.

left inferior and middle temporal gyrus; (3) the left supramarginal gyrus; (4) the precuneus; (5) the right inferior frontal gyrus (pars triangularis) and frontal operculum; (6) the left supramarginal gyrus; and (7) the left middle frontal.

During the rest condition, consciousness-level-dependent increases of intrinsic connectivity were found in two clusters covering (1) the left middle temporal gyrus and (2) precuneus (Fig. 3a and Supplementary Table S4).
Looking at effect sizes of each condition separately, we observe the same pattern that was previously observed in the network-to-voxel analyses of rest and music conditions alone. The connectivity increases along with the level of consciousness from (E)MCS to HCS, while the group of UWS presents a hyperconnectivity (Supplementary Table S4 and Supplementary Fig. S5).

No significant results were found when the music condition was compared with the rest condition.

Amplitude of low-frequency fluctuation. During the music condition, consciousness-level-dependent increases in ALFF were found in nine clusters (Fig. 3b and Supplementary Table S4) covering (1) the posterior cingulate gyrus, (2) the precuneus, (3) the left frontal pole, (4) the left planum polare and temporal pole, (5) the left inferior temporal gyrus, (6) the left parahippocampal gyrus, (7) the left middle temporal gyrus, (8) the brainstem, and (9) the left inferior temporal gyrus.

During the rest condition, consciousness-level-dependent increases in ALFF were observed in four clusters covering (1) the left posterior cingulate gyrus, (2) the left frontal pole, (3) the right supramarginal gyrus, and (4) the left frontal pole (Fig. 3b and Supplementary Table S4).

Looking at the effect sizes of both conditions separately, a linear gradation of the activity along with the level of consciousness (from (E)MCS to HCS) is once again observed for all the clusters, while a hyperactivity is observed for UWS patients.

When comparing the music condition with the rest condition, consciousness-level-dependent increases in ALLF were observed in nine clusters covering (1) the brainstem, (2) the vermis, (3) the right temporal pole, (4) the brainstem, (5) the left inferior and middle temporal gyrus, (6) the left angular gyrus, (7) the left posterior inferior temporal gyrus, (8) the left temporal pole and insular cortex, and (9) the left middle temporal gyrus (Fig. 3b and Supplementary Table S4). Looking at the effect sizes, we see that the (E)MCS and LIS groups are the one driving the changes in ALFF between the two conditions. Across most of the clusters, (E)MCS patients either stay constant or have decreased ALFF, while LIS patients either stay constant or have increased ALFF (Fig. 4).

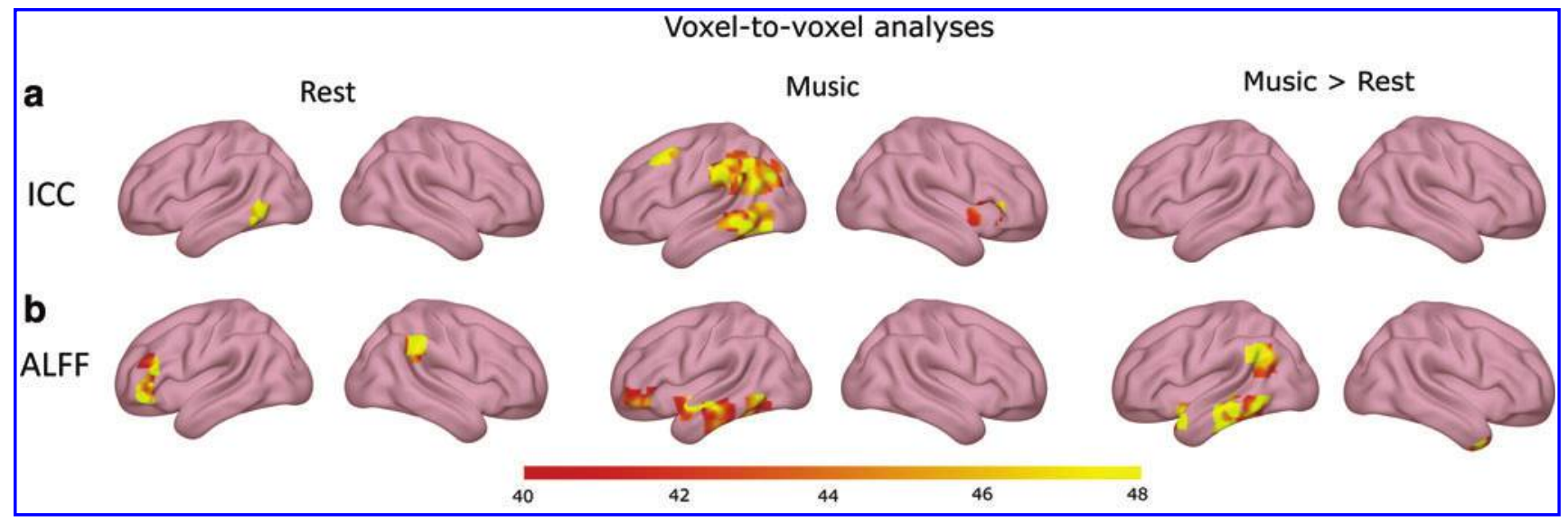

FIG. 3. Voxel-to-voxel analysis of the ICC (a) and of the ALFF (b). The maps show brain areas with stronger consciousness-level-dependent increases during the rest condition (on the left), the music condition (in the middle), and during the music compared with the rest condition (on the right). ALFF, amplitude of low-frequency fluctuation; ICC, intrinsic connectivity contrast. Color images are available online. 


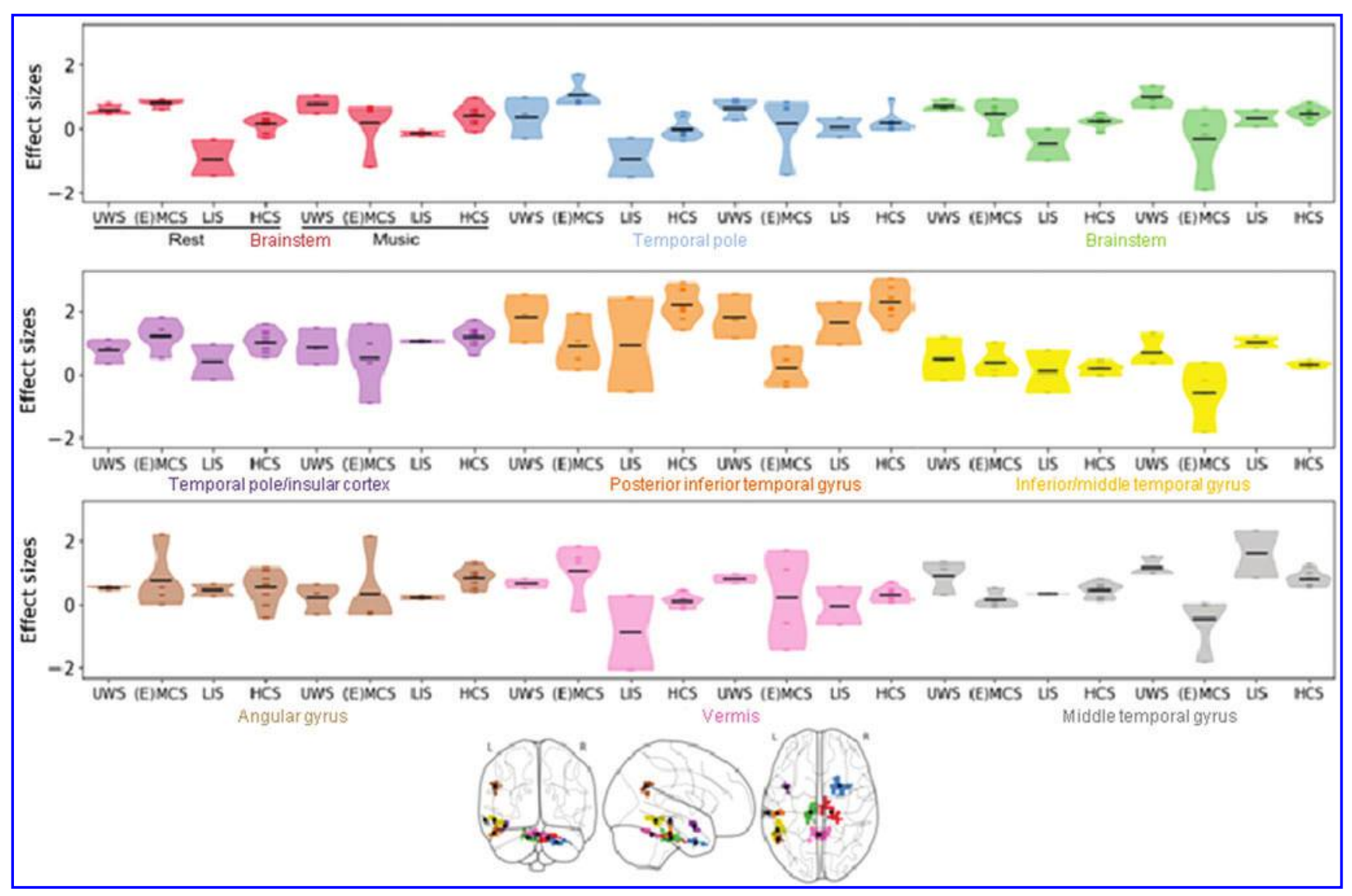

FIG. 4. Effect sizes ( $z$-values) for the clusters showing increased ALFF (activity) in the music compared with the rest condition at the whole brain level. The violin plots represent kernel density estimation. Each dot represents one patient/healthy subject and black lines represent the means. Each color represents one cluster. Color images are available online.

No consciousness-level-dependent increases in fALFF were found.

\section{Discussion}

In this study, we explored the effects of preferred music on brain functional connectivity in relation to consciousness. Among the four investigated networks (network-to-voxel analysis), the FPN (also known as task-positive or external network) was the only one showing stronger consciousnesslevel-dependent increases of connectivity in the music compared with the rest condition. This stronger relationship to consciousness during preferred music was found in the precuneus, which is known to be especially important in internal awareness (Stewart et al., 2006) as well as episodic memory (Fletcher et al., 1995). The precuneus also plays a key role in music processing and visual imagery (Nakamura et al., 1999).

While this study could not find any consciousness-related functional connectivity changes for the FPN in the rest condition alone (contrary to previous studies: Demertzi et al., 2014, 2015), several regions showed connectivity increases during the music condition. During music, connectivity with the left angular gyrus, the frontal pole, and the right middle frontal gyrus showed a relation to consciousness. The angular gyrus is well known for its role in episodic memory, and may therefore intervene in the recognition of music and the recall of personal associated memories evoked by music (Bonnici et al., 2018). In healthy subjects, for example, the angular gyrus showed increased functional connectivity during an imagined music performance task (Tanaka and Kirino, 2019). In addition, this region was shown to be involved in language processing in patients with DOC (Aubinet et al., 2020), which might be related to the processing of lyrics. Moreover, right frontal activity has previously been related to the processing of mode and tempo (Khalfa et al., 2005), and is strongly associated with emotional processing (Bidelman, 2013). Overall, the regions showing consciousness-level-related increases in connectivity during music have previously been observed to relate to consciousness in larger studies within classical rest conditions (Demertzi et al., 2015).

Functional connectivity with the DMN showed a relation to the level of consciousness in both the rest and music conditions, although no significant difference was found between the two conditions. Both conditions individually showed increased connectivity in the precuneus and parts of the posterior cingulate gyrus. In the rest condition, connectivity increases were also observed in the left hippocampus and right middle temporal gyrus. These results are consistent with previous findings of increased DMN connectivity (Vanhaudenhuyse et al., 2010) and decreased ICC and entropy (Luppi et al., 2019) in proportion to the level of 
consciousness in brain-injured patients. Furthermore, several regions showed increased connectivity only in the music condition: the left lateral occipital cortex and the left inferior frontal gyrus. These regions have all been related to musical processing; connectivity of the lateral occipital cortex and precuneus is related to musical training (Tanaka and Kirino, 2016), while the inferior frontal gyrus is involved in the recognition of familiar melodies (Satoh et al., 2006).

Whole brain functional connectivity results (ICC) are in line with those of the network-level analysis mentioned above. The music and rest conditions independently showed significant connectivity increases as a function of the level of consciousness in the middle temporal gyrus and precuneus. In the music condition, stronger connectivity as a function of consciousness was also observed in multiple regions already highlighted in the network-to-voxel analyses, namely those involved in language and semantic processing (i.e., the left angular gyrus, left supramarginal gyrus, left lateral occipital cortex, left inferior temporal gyrus) (Visser et al., 2010). Besides these areas, the right inferior frontal gyrus and the right frontal operculum were found to have stronger connectivity as a function of consciousness in the music condition. These two regions are known to be involved in music processing, respectively, in the perception of the emotional content of music (Tabei, 2015), and in pitch processing and melody generation (Stewart et al., 2006).

In addition to the investigation of functional connectivity, we probed the potential positive changes that music could induce on the magnitude of brain regional spontaneous activity. When comparing music with rest, we found increasing ALFF (but not fALFF) in the brainstem, the vermis, the temporal pole, the left inferior and middle temporal gyrus, left insular cortex and the left angular gyrus, all of which are music-related brain areas. For example, the cerebellar vermis and the brainstem are important in musical temporal structure processing (Levitin, 2009; Bidelman, 2013), the insular cortex is involved in pleasant and familiar music processing (Stewart et al., 2006; Koelsch, 2014), the middle temporal gyrus is known to play a role in auditory and linguistic processing, as well as music memory (Binder et al., 1996; Watanabe et al., 2008), and the inferior temporal gyrus is involved in stimulus recognition (Buckley et al., 1997).

Similarly to the connectivity analysis, both the rest and music conditions individually showed regions of activation in relation to consciousness (Fig. 3 and Supplementary Table S4). Furthermore, consciousness-level-dependent changes in activity are again found more substantial during the music condition than during the rest condition. In line with the above-mentioned results, all regions of increased activity can be related to musical processing. On the contrary, the absence of significant results with fALFF must urge us to consider the results from ALFF with the greatest caution (Mao et al., 2015). For this reason, future studies should replicate these results to investigate if the presence of results with ALFF but not with fALFF might imply that noise is being measured instead of the signal.

With a partial reanalysis of patients' data (Heine et al., 2015), we provide further relevant insights on the importance of preferred stimuli during assessment of patients' cognitive capacity. The results converge furthermore with previous studies, and provide evidence that music might potentially trigger activity and connectivity changes more substantially compared with a rest condition without any sensory stimulation. These effects are mostly observed in brain regions involved in consciousness, episodic memory, but also in auditory, language, and emotion processing.

Moreover, the effect sizes of the significant results provide us additional insights, showing that the difference in connectivity observed between the two conditions in the FPN (with the precuneus) is mostly driven by a decrease of connectivity in (E)MCS patients. We expect this observed decrease in connectivity to be due to stronger anticorrelations in EMCS patients. Previous research has shown that anticorrelations are observable with the FPN in EMCS patients, but not in MCS and UWS (Di Perri et al., 2016). However, the observed effects could also be due to a decrease of positive connectivity or even a reorganization of cerebral networks. Regarding ALFF, a music-induced effect is also identifiable, with a wide decrease in activity in several regions observed in (E)MCS and an increase in LIS patients.

Although these findings have to be taken and interpreted with caution due to our small sample size, we did not find any substantial changes in UWS and HCS in terms of connectivity or activity due to preferred music. These effects of music therefore could only be observed in severely brain-injured patients with some preserved awareness. The absence of effect in HCS is in line with the findings of two previous studies (Castro et al., 2015; Heine et al., 2015). To explain the absence of effect in HCS, these studies hypothesized that the cerebral responses of the HCS cannot be enhanced because they are already at ceiling, or because of the control condition, which would be too neutral to observe a difference with the music condition. Alternatively, it is possible that the effect of music on HCS may be more subtle than the response evoked in brain-injured patients (who experience a reduction of sources of stimulations due to their condition, and hence may be more sensitive to stimuli such as music), which would require a bigger sample size to observe.

Finally, in all our results, the group of UWS seems to present a constant hyperconnectivity/activity, whether during the music or the rest condition. In the absence of any difference between the two conditions, we can conclude that music does not seem to trigger any stronger effect on UWS patients. Even if this pattern is surprising, it is however not without precedent since other studies also evidenced hyperconnectivity in these patients (Di Perri et al., 2013, 2016; Wu et al., 2015). One hypothesis would be a persistent engagement of residual neural activity in self-reinforcing neural loops, which, in turn, might disrupt normal patterns of connectivity.

Our study has several limitations. Due to the experiment design, only patients who were not sedated, remained calm enough, and had close relatives who knew their favorite music could be included, leading to a small sample size. The low number of patients and its heterogeneity prevent us from making strong assumptions or generalizing the findings. However, the obtained results are in line with previous studies on music interventions in DOC (Grimm and Kreutz, 2018). Moreover, our sample was divided into four groups: unconsciousness, fluctuating consciousness, LIS, and normal consciousness. Further studies should therefore focus on exploring potential effects within the minimally conscious population.

Furthermore, we decided to include LIS patients in our gradient of consciousness, placing them between MCS- 
EMCS and HCS. Although, by definition, LIS have relatively preserved cognitive capabilities, our two LIS patients presented below-standard performance in neuropsychological tests evaluating visual memory, executive functions, language, and sustained attention. We therefore postulated that they could present stronger music-related effects, compared with HCS, which generally show no effect of music (Castro et al., 2015; Heine et al., 2015).

Finally, the study employs preferred music because of the beneficial effects of preferred music demonstrated in other pathologies such as stroke (Soto et al., 2009), and those of salient stimuli on DOC patients. We assumed preference can maximize the effect of music on brain function. However, it is entirely possible that the observed effects are not due to the preference itself and we cannot draw conclusions as to which properties of (preferred) music we can attribute the effects. Even though the chosen music was vastly different within and between subjects, the results could indeed be due to the structural and acoustic features of the musical pieces. Furthermore, our findings are in line with both of the existing theories explaining the beneficial effect of music at present, namely the "Mood and arousal hypothesis" (Nantais and Schellenberg, 1999), and the autobiographical priming (Castro et al., 2015). Further research should focus on effects of music in general and its specific acoustic features as well as simple auditory stimuli. Future studies should also explore the effect of preference to distinguish between the current theories. In this context, the exploration of the salience network might also be of interest.

In conclusion, music seems to enhance brain functioning by strengthening the connectivity and increasing regional spontaneous activity of a wide range of subcortical and cortical brain regions in a conscious-level-dependent manner. These results constitute an indication of stronger musical processing in higher states of consciousness. They could also point toward the ability of music to temporarily stimulate patients' brain functions. Overall, although our results should be taken with caution given the small sample size and the heterogeneity of patients ' clinical condition, they seem to be in favor of the hypothesis that music could play a role in improvements of diagnostic assessment.

\section{Authors' Contributions}

L.H., F.P., C.M., and M.C. conceived, planned, and conducted the research and analyses. M.C., S.K.L., L.H., and C.M. interpreted the results. S.K.L. and M.A.B. designed the methods and scripts for data preprocessing and analysis on fMRI. M.C. and S.K.L realized the visualizations. L.H., M.C., C.A., and C.M. carried out the data acquisitions. C.M. and L.H. provided guidance and supervision on the whole study. S.L. provided supervision and resources. M.C., S.K.L., and L.H. drafted the article, and all authors provided critical feedback and helped shape the final article. S.K.L and C.A. are FRS-FNRS research fellows, and S.L. is research director at FRS-FNRS.

\section{Acknowledgments}

The authors thank the patients and their families for participating in this study, as well as Dr. J.-F.L. Tshibanda, Mr. J. Klein, Mrs. N. Maquet, Mr. J.-M. Léonard, and the whole staff from the Neurology, Radiodiagnostic, and
Nuclear Medicine departments, University Hospital of Liège, the Cyclotron of the University of Liège, the Siemens Healthineers, and Prof. A. Nieto-Castanon of the Department of Speech, Language, and Hearing sciences, Boston University (USA) for their kind technical support and advices. They also thank the reviewers who provided excellent feedback.

\section{Author Disclosure Statement}

No competing financial interests exist.

\section{Funding Information}

This research was supported by the University and University Hospital of Liège, the Belgian National Funds for Scientific Research (FRS-FNRS), the European Union's Horizon 2020 Framework Programme for Research and Innovation under the Specific Grant Agreement No. 785907 (Human Brain Project SGA2), the Luminous project (EU-H2020-fetopenga686764), CogniComa (ANR-14-CE-15-0013), the LabEx CelyA (ANR-10-LABX-0060/ANR-16-IDEX-0005) the European Space Agency (ESA) and the Belgian Federal Science Policy Office (BELSPO) in the framework of the PRODEX Programme, the Center-TBI project (FP7HEALTH-602150), the Public Utility Foundation 'Université Européenne du Travail", "Fondazione Europea di Ricerca Biomedica," the Bial Foundation, the Mind Science Foundation and the European Commission.

\section{Supplementary Material}

Supplementary Table S1

Supplementary Table S2

Supplementary Table S3

Supplementary Table S4

Supplementary Figure S1

Supplementary Figure S2

Supplementary Figure S3

Supplementary Figure S4

Supplementary Figure S5

\section{References}

Aubinet C, Cassol H, Gosseries O, Bahri MA, Larroque SK, Majerus S, et al. 2020. Brain metabolism but not grey matter volume underlies the presence of language function in the minimally conscious state (MCS): MCS+ versus MCS- neuroimaging differences. Neurorehabil Neural Repair 34:172184.

Behzadi Y, Restom K, Liau J, Liu TT. 2007. A component based noise correction method (CompCor) for BOLD and perfusion based fMRI. Neuroimage 37:90-101.

Bidelman GM. 2013. The role of the auditory brainstem in processing musically relevant pitch. Front Psychol 4:1-13.

Binder JR, Frost JA, Hammeke TA, Rao SM, Cox RW. 1996. Function of the left planum temporale in auditory and linguistic processing. Brain 119:1239-1247.

Boly M, Faymonville ME, Schnakers C, Peigneux P, Lambermont B, Phillips C, et al. 2008. Perception of pain in the minimally conscious state with PET activation: an observational study. Lancet Neurol 7:1013-1020.

Bonnici HM, Cheke LG, Green DAE, FitzGerald THMB, Simons JS. 2018. Specifying a causal role for angular gyrus in autobiographical memory. J Neurosci 38: 10438-10443. 
Buckley MJ, Gaffan D, Murray EA. 1997. Functional double dissociation between two inferior temporal cortical areas: perirhinal cortex versus middle temporal gyrus. J Neurophysiol 77:587-598.

Castro M, Tillmann B, Luaute J, Corneyllie A, Dailler F, AndreObadia N, et al. 2015. Boosting cognition with music in patients with disorders of consciousness. Neurorehabil Neural Repair 29:734-742.

Cheng L, Gosseries O, Ying L, Hu X, Yu D, Gao H, et al. 2013. Assessment of localisation to auditory stimulation in postcomatose states: use the patient's own name. BMC Neurol $13: 27$.

Demertzi A, Antonopoulos G, Heine L, Voss HU, Crone JS, De Los Angeles C, et al. 2015. Intrinsic functional connectivity differentiates minimally conscious from unresponsive patients. Brain 138:2619-2631.

Demertzi A, Gómez F, Crone JS, Vanhaudenhuyse A, Tshibanda L, Noirhomme Q, et al. 2014. Multiple fMRI system-level baseline connectivity is disrupted in patients with consciousness alterations. Cortex 52:35-46.

Demertzi A, Ledoux D, Bruno MA, Vanhaudenhuyse A, Gosseries O, Soddu A, et al. 2011. Attitudes towards end-of-life issues in disorders of consciousness: A European survey. $\mathbf{J}$ Neurol 258: 1058-1065.

Di Perri C, Bahri MA, Amico E, Thibaut A, Heine L, Antonopoulos G, et al. 2016. Neural correlates of consciousness in patients who have emerged from a minimally conscious state: a cross-sectional multimodal imaging study. Lancet Neurol 15:830-842.

Di Perri C, Bastianello S, Bartsch AJ, Pistarini C, Maggioni G, Magrassi L, et al. 2013. Limbic hyperconnectivity in the vegetative state. Neurology 81:1417-1424.

Fletcher P, Frith C, Baker S, Shallice T, Frackowiak RS, Dolan R. 1995. The mind's eye-precuneus activation in memoryrelated imagery. Neuroimage 2:195-200.

Giacino JT, Ashwal S, Childs N, Cranford R, Jennett B, Katz DI, et al. 2002. The minimally conscious state: definition and diagnostic criteria. Neurology 58:349-353.

Giacino JT, Kalmar K, Whyte J. 2004. The JFK coma recovery scale-revised: measurement characteristics and diagnostic utility. Arch Phys Med Rehabil 85:2020-2029.

Grimm T, Kreutz G. 2018. Music interventions in disorders of consciousness (DOC) - a systematic review. Brain Inj 32: 704-714.

Heine L, Castro M, Martial C, Tillmann B, Laureys S, Perrin F. 2015. Exploration of functional connectivity during preferred music stimulation in patients with disorders of consciousness. Front Psychol 6:1-11.

Heine L, Soddu A, Gómez F, Vanhaudenhuyse A, Tshibanda L, Thonnard M, et al. 2012. Resting state networks and consciousness Alterations of multiple resting state network connectivity in physiological, pharmacological, and pathological consciousness states. Front Psychol 3:1-12.

Khalfa S, Schon D, Anton JL, Liégeois-Chauvel C. 2005. Brain regions involved in the recognition of happiness and sadness in music. Neuroreport 16:1981-1984.

Koelsch S. 2014. Brain correlates of music-evoked emotions. Nat Rev Neurosci 15:170-180.

Levitin DJ. 2009. The neural correlates of temporal structure in music. Music Med 1:9-13.

Luppi AI, Craig MM, Finoia P, Williams GB, Naci L, Menon DK, et al. 2019. Consciousness-specific dynamic interactions of brain integration and functional diversity. Nat Commun 10:1-12.
Magee WL. 2005. Music therapy with patients in low awareness states: approaches to assessment and treatment in multidisciplinary care. Neuropsychol Rehabil 15:522536.

Mao D, Ding Z, Jia W, Liao W, Li X, Huang H, et al. 2015. Lowfrequency fluctuations of the resting brain: high magnitude does not equal high reliability. PLoS One 10:1-20.

Nakamura S, Sadato N, Oohashi T, Nishina E, Fuwamoto Y, Yonekura Y. 1999. Analysis of music-brain interaction with simultaneous measurement of regional cerebral blood flow and electroencephalogram beta rhythm in human subjects. Neurosci Lett 275:222-226.

Nantais KM, Schellenberg EG. 1999. The mozart effect: an artifact of preference. Psychol Sci 10:370-373.

Okumura Y, Asano Y, Takenaka S, Fukuyama S, Yonezawa S, Kasuya Y, et al. 2014. Brain activation by music in patients in a vegetative or minimally conscious state following diffuse brain injury. Brain Inj 9052:1-7.

Perrin F, Castro M, Tillmann B, Luauté J. 2015. Promoting the use of personally relevant stimuli for investigating patients with disorders of consciousness. Front Psychol 6:1-9.

Raglio A, Guizzetti GB, Bolognesi M, Antonaci D, Granieri E, Baiardi P, et al. 2014. Active music therapy approach in disorders of consciousness: a controlled observational case series. J Neurol 261:2460-2462.

Satoh M, Takeda K, Nagata K, Shimosegawa E, Kuzuhara S. 2006. Positron-emission tomography of brain regions activated by recognition of familiar music. Am J Neuroradiol 27:1101-1106.

Schiff ND. 2015. Cognitive motor dissociation following severe brain injuries. JAMA Neurol 72:1413-1415.

Schnakers C, Majerus S, Goldman S, Boly M, Van Eeckhout P, Gay S, et al. 2008. Cognitive function in the locked-in syndrome. J Neurol 255:323-330.

Schnakers C, Vanhaudenhuyse A, Giacino J, Ventura M, Boly M, Majerus S, et al. 2009. Diagnostic accuracy of the vegetative and minimally conscious state: clinical consensus versus standardized neurobehavioral assessment. BMC Neurol 9:1-5.

Soto D, Funes MJ, Guzmán-García A, Warbrick T, Rotshtein P, Humphreys GW. 2009. Pleasant music overcomes the loss of awareness in patients with visual neglect. Proc Natl Acad Sci U S A 106:6011-6016.

Statistical Parametric Mapping 12. www.fil.ion.ucl.ac.uk/spm/ software/spm12 Last accessed December 10, 2019.

Stewart L, Von Kriegstein K, Warren JD, Griffiths TD. 2006. Music and the brain: disorders of musical listening. Brain 129:2533-2553.

Sun Y, Wang J, Heine L, Huang W, Wang J, Hu N, et al. 2018. Personalized objects can optimize the diagnosis of EMCS in the assessment of functional object use in the CRS-R: a double blind, randomized clinical trial. BMC Neurol 18:1-5.

Tabei K. 2015. Inferior frontal gyrus activation underlies the perception of emotions, while precuneus activation underlies the feeling of emotions during music listening. Behav Neurol 2015:1-6.

Tanaka S, Kirino E. 2016. Functional connectivity of the precuneus in female university students with long-term musical training. Front Hum Neurosci 10:1-7.

Tanaka S, Kirino E. 2019. Increased functional connectivity of the angular gyrus during imagined music performance. Front Hum Neurosci 13: 1-8.

The Multi-Society Task Force on PVS. 1994. Medical aspects of the persistent vegetative state. N Engl J Med 330:1499-1508. 
Turner JA, Damaraju E, Van Erp TGM, Mathalon DH, Ford JM, Voyvodic J, et al. 2013. A multi-site resting state fMRI study on the amplitude of low frequency fluctuations in schizophrenia. Front Neurosci 7:1-13.

Vanhaudenhuyse A, Noirhomme Q, Tshibanda LJF, Bruno MA, Boveroux P, Schnakers C, et al. 2010. Default network connectivity reflects the level of consciousness in noncommunicative brain-damaged patients. Brain 133:161-171.

Verger J, Ruiz S, Tillmann B, Ben Romdhane M, De Quelen M, Castro M, et al. 2014. Beneficial effect of preferred music on cognitive functions in minimally conscious state patients. Rev Neurol (Paris) 170:693-699.

Visser M, Embleton KV, Jefferies E, Parker GJ, Ralph MAL. 2010. The inferior, anterior temporal lobes and semantic memory clarified: novel evidence from distortion-corrected fMRI. Neuropsychologia 48:1689-1696.

Wannez S, Heine L, Thonnard M, Gosseries O, Laureys S. 2017a. The repetition of behavioral assessments in diagnosis of disorders of consciousness. Ann Neurol 81: 883-889.

Wannez S, Vanhaudenhuyse A, Laureys S, Brédart S. 2017 b. Mirror efficiency in the assessment of visual pursuit in patients in minimally conscious state. Brain Inj 31:1429-1435.
Watanabe T, Yagishita S, Kikyo H. 2008. Memory of music: roles of right hippocampus and left inferior frontal gyrus. Neuroimage 39:483-491.

Whitfield-Gabrieli S, Nieto-Castanon A. 2012. Conn: a functional connectivity toolbox for correlated and anticorrelated brain networks. Brain Connect 2:125-141.

Wu X, Zou Q, Hu J, Tang W, Mao Y, Gao L, et al. 2015. Intrinsic functional connectivity patterns predict consciousness level and recovery outcome in acquired brain injury. $\mathrm{J}$ Neurosci 35:12932-12946.

Address correspondence to: Steven Laureys Coma Science Group, GIGA-Consciousness University of Liège University Hospital of Liège Centre du Cerveau2, Avenue de l'hôpital 1

Liège 4000 Belgium

E-mail: steven.laureys@uliege.be 